\title{
Vehicle routing problem with cross-docking in a sustainable supply chain for perishable products
}

Fatemeh Shahrabi ( $\square$ fshahrabi67@ut.ac.ir)

University of Tehran https://orcid.org/0000-0002-6443-4858

Mohammad Mahdi Nasiri

University of Tehran https://orcid.org/0000-0001-9813-1233

S. Mohammad J. Mirzapour Al-e-Hashem

Rennes School of Business France: Rennes School of Business

\section{Research Article}

Keywords: Sustainable vehicle routing problem, Cross-docking, Freshness, Job satisfaction, Genetic algorithm, Social responsibility

Posted Date: February 28th, 2022

DOI: https://doi.org/10.21203/rs.3.rs-1220987/v1

License: @ (i) This work is licensed under a Creative Commons Attribution 4.0 International License. Read Full License 


\title{
Vehicle routing problem with cross-docking in a sustainable supply chain for perishable products
}

\author{
Fatemeh Shahrabi a , Mohammad Mahdi Nasiri ${ }^{a,}{ }^{*}$, S. Mohammad J. Mirzapour Al-e-Hashem ${ }^{\text {b }}$ \\ a School of Industrial Engineering, College of Engineering, University of Tehran, Tehran, Iran \\ ${ }^{b}$ Rennes School of Business, 2 Rue Robert d'Arbrissel, 35065 Rennes, France
}

\begin{abstract}
Today's transportation, which is primarily based on the combustion of fossil fuels, contributes significantly to energy-related GHG emissions which is one of aspects of sustainability. Incorporating sustainable concerns in cross-dock scheduling can affect decision making for logistics systems. In the current contribution, a sustainable vehicle routing problem with cross-docking is presented. In addition to the economic considerations, this paper addresses the environmental impacts of $\mathrm{CO} 2$ emissions and the social impacts, including equity between drivers as well as customer satisfaction. A metaheuristic, composed of GA and mixed integer programming, is proposed as the solution approach. For validating the presented method, instances of various sizes are solved. The results of the GA in small-sized instances, has a small deviation from the optimal fitness values. Finally, a real case study is provided to demonstrate the applicability of the model in a real-world environment, and several in-depth analyses are conducted to infer managerial implications.
\end{abstract}

Keywords: Sustainable vehicle routing problem, Cross-docking, Freshness, Job satisfaction, Genetic algorithm, Social responsibility

\section{Introduction}

Cross-docking is a distribution strategy for logistics companies which aim to decrease the delivery time and the storage cost. In a typical cross-dock facility, products are unloaded from incoming vehicles which carry products from suppliers, at the receiving doors, sorted according to the orders of customers, stored and staged, and then, loaded into outgoing vehicles at shipping doors to be delivered to customers (Chargui et al., 2020). The time spent by the products in a cross-dock facility typically do not exceed $24 \mathrm{~h}$ (Arbabi et al., 2021). To achieve an optimized delivery, the cross-dock scheduling should be considered together with the routing of outgoing vehicles in a vehicle routing problem with cross-docking (VRPCD). For a comprehensive review of VRPCD papers, see Nasiri et al. (2018).

\footnotetext{
* Corresponding author Email address: mmnasiri@ut.ac.ir
} 
According to the Intergovernmental Panel on Climate Change's fifth assessment report, in order to keep warming below 2 degrees Celsius, GHG emissions must be reduced by 40 to 70 percent by 2050, compared to 2010 levels (IPCC, 2014). As a result, Governments are under increasing pressure to enact legislation to reduce the volume of these emissions (Benjaafar et al., 2012). One of the major drivers to GHG emissions has been identified as logistics and supply chain operations.

For many years, minimizing the total cost or maximizing the profit were the main objectives of crossdocking facility managers, but today, they are also responsible for other dimensions of a sustainable development (i.e., the environmental and social aspects). Brundland (1987) defined sustainability as: "the ability to meet the needs of the present without compromising the ability of future generations to meet their own needs". In the next two subsections, we investigate the works which are related to VRPCD, and consider sustainability, or at least the environmental aspect. 


\subsection{Sustainable cross-docking}

There are few sustainable and green studies in this scope. Yin et al. (2016) investigated green VRPCD considering constraints on the amount of $\mathrm{CO}_{2}$ emissions. Yin and Chuang (2016) tried to reach an efficient fuel consumption through cost minimization and $\mathrm{CO}_{2}$ emissions. Govindan et al. (2015) considered sustainable order allocation and sustainable supply chain through two objectives. The first objective is to minimize related costs and the second objective is to minimize the environmental impact of all the members of a supply chain and open/established plants, distribution centers, and cross-docks are taken into account. Abad et al. (2018) studied a pollution routing problem with a cross-docking system.

Rezaei and Kheirkhah (2018) considered all the three aspects of sustainable development in a cross-dock related problem. Our work is different from Rezaei and Kheirkhah (2018), as they focus on strategic level decisions in a network design framework, and do not take the effect of operational decisions into account. In addition, their social and environmental criteria are different from those of our research. Furthermore, Tirkolaee et al. (2020) proposed reliability in a pollution routing problem with cross dock selection as customer satisfaction which is considered in the social dimension. The current research is different from the one of Tirkolaee et al. (2020) which considered the strategic decisions of the supply chain.

\subsection{Sustainable VRP including social aspect}

Many authors concentrate on the environmental aspect in their studies, and overlook the social aspect. Noise pollution is one of the social factors which has an impact on quality of life and health. Ćirović et al. (2014) limited traffic noise level in the road which is affected by a number of passenger, number of heavy vehicles and the number of buses. In addition, Rahimi et al. (2016) considered noise level as a constraint which depends on the route and type of vehicles. Furthermore, Bandeira et al. (2018) formulated the varying speed due to acceleration and deceleration as an important factor for calculating the noise level.

When the drivers have to wait a long time in the congestion, it leads to the job dissatisfaction. Zhu and $\mathrm{Hu}$ (2019) investigated a sustainable routing problem in a congestion situation. In order to avoid heavy congestions, they took the waiting time of the drivers into account at the customer's nodes and route.

For strategic level, career opportunity is one of the social impacts. Zhalechian et al. (2016) discussed the employment level in a location routing problem. Equity among the drivers is studied by Ramos et al. (2014) as another social goal, which helps overloaded drivers to work less than before. Furthermore, Govindan et al. (2019) investigated job opportunities in a sustainable supply chain with the vehicle routing problem. Also, Shahedi et al. (2021) considered employments opportunity which is created by the establishment of the required facilities.

Accident prevention is another social impact in routing problem. Rahimi et al. (2016) considered accident rate proportional average traffic speed and vehicle speed in the selected route. Also, Reyes-Rubiano et al. (2018) studied vehicle load and distance traveled in accident risk. Bandeira et al. (2018) presented a safety model based 
on empirical GPS data and microscopic simulation. Abdullahi et al. (2021) estimated the vehicle accident according to their load and travel distance. Furthermore, Peng et al. (2020) estimated the likelihood of the accident on each route considering the social impact of the load and the type of the vehicle. They believe that the social impact of a fully loaded truck is more than an empty one when an accident occurs.

Customer satisfaction is the most frequently studied criterion of the social dimension (Table 1). Freshness of products and food quality, minimization of food waste, and on-time delivery are major factors that the customer satisfaction is depended on.

Table 1 shows recent studies on the sustainable VRP that take the social aspect into account. It should be noted that some researchers investigated economic as well as social aspects, but environmental consideration is neglected. So, we only present researches with all the three dimensions.

Table 1. New researches sustainable VRP with social aspect

\begin{tabular}{|c|c|c|c|c|c|c|}
\hline \multirow[b]{2}{*}{ Research } & \multicolumn{4}{|c|}{ Job satisfaction } & \multirow{2}{*}{$\begin{array}{c}\text { Noise } \\
\text { emission }\end{array}$} & \multirow[b]{2}{*}{$\begin{array}{l}\text { Customer } \\
\text { satisfaction }\end{array}$} \\
\hline & $\begin{array}{c}\text { Driver's } \\
\text { waiting } \\
\text { time }\end{array}$ & $\begin{array}{c}\text { Career } \\
\text { opportunities }\end{array}$ & $\begin{array}{l}\text { Accident } \\
\text { prevention }\end{array}$ & $\begin{array}{c}\text { Equity } \\
\text { between } \\
\text { drivers }\end{array}$ & & \\
\hline Ćirović et al. (2014) & & & & & $\checkmark$ & \\
\hline Ramos et al. (2014) & & & & $\checkmark$ & & \\
\hline Afshar-Bakeshloo et al. (2016) & & & & & & $\checkmark$ \\
\hline Rahimi et al. (2016) & & & $\checkmark$ & & $\checkmark$ & $\checkmark$ \\
\hline Song and Ko (2016) & & & & & & $\checkmark$ \\
\hline Tavakkoli-Moghaddam and Raziei (2016) & & & & & & $\checkmark$ \\
\hline Zhalechian et al. (2016) & & $\checkmark$ & & & & \\
\hline Hosseini-Nasab and Lotfalian (2017) & & & & & & $\checkmark$ \\
\hline Bandeira et al. (2018) & & & $\checkmark$ & & $\checkmark$ & \\
\hline Reyes-Rubiano et al. (2018) & & & $\checkmark$ & & & \\
\hline Govindan et al. (2019) & & $\checkmark$ & & & & $\checkmark$ \\
\hline Zhu and $\mathrm{Hu}$ (2019) & $\checkmark$ & & & & & \\
\hline Chan et al. (2020) & & & & & & $\checkmark$ \\
\hline Peng et al. (2020) & & & $\checkmark$ & & & \\
\hline Qiao et al. (2020) & & & & & & $\checkmark$ \\
\hline Alamatsaz et al. (2021) & & & & & & $\checkmark$ \\
\hline Abdullahi et al. (2021) & & & $\checkmark$ & & & \\
\hline Shahedi et al. (2021) & & $\checkmark$ & & & & \\
\hline Shahrabi et al. (2021) & & & & & & $\checkmark$ \\
\hline This study & & & & $\checkmark$ & & 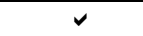 \\
\hline
\end{tabular}

\subsection{Contributions of this paper}

The studies reviewed so far reveal some research gaps. Most of the works that are interested in the sustainable VRP area have only focused on the economic and green aspects of sustainability, and social factors are missing. In addition, previous studies have not dealt with an operational model for a sustainable cross-dock. To fulfill this shortcoming, this paper presents a new sustainable VRP model using cross-dock facility incorporating social concerns. Our main contributions that distinguish our efforts from the related studies are:

- including all the three aspects of sustainability to have a complete foundation of this concept in a cross-dock in the operational level, as routing problem decisions are operational decisions; and

- obtaining a fair cross-dock schedule for drivers by balancing their working hours to satisfy them as a social responsibility concern. 
The proposed model optimizes the decisions in a cross-docking center, i.e., scheduling and sequencing of incoming vehicles at receiving doors, scheduling and sequencing of outgoing vehicles at shipping doors and delivery routing to achieve fair working hours for drivers. 


\subsection{Outline}

The reminder of this paper is as follows. In Section 2, the problem is described and the mixed-integer linear programming (MILP) model is presented through objective function and constraints. Section 3 is devoted to the solution approach. The experimental results and case study are presented in Section 4. Finally, in Section 5 the main and overall conclusions and also the offers for future researches are described.

\section{Problem description, notations, and mathematical model}

This section develops a model for making optimal decisions about a cross-dock which distributes orders of customers. The cross-dock has $F$ receiving dock doors indexed by $f \in\{1, \ldots, F\}$ which serve a set of $L$ incoming vehicles indexed by $l \in\{1, \ldots, L\}$, and $H$ shipping dock doors indexed by $h \in\{1, \ldots, H\}$ which serve a set of $K$ outgoing vehicle indexed by $k \in\{1, \ldots, K\}$. In addition, there are $n$ customers (nodes) indexed by $i \in N=$ $\{1, \ldots, n\}$, and $P$ product types indexed by $p \in\{1, \ldots, P\}$. Customer $i$ has a certain demand of $D_{i p}$ units of product type $p$, which should be met. The orders of customers are carried by incoming vehicles to the cross-dock parking yard. Next, it takes $t t$ minutes for the incoming vehicle to reach the dock door. It also takes $P U_{i l}$ minutes to unload the order of customer $i$ shipped by incoming vehicle $l$. Then, the orders are transferred from the receiving dock door to one of the shipping dock doors which takes TRS minutes. After that, the order of customer $i$ is loaded on outgoing vehicle $k$ at the shipping dock door, and the process takes $P L_{i k}$ minutes. At the beginning of the planning horizon, all of the outgoing vehicles are available at the shipping dock doors. In addition, we are looking for a sustainable routing for outgoing vehicles to leave the cross-dock (indexed by 0 and $n+1$ ), visit $n$ customers (nodes) and deliver the orders. Each customer visited by only one outgoing vehicle and after delivering orders to the customer $j$ in $S T_{j}$ minutes they return to cross-dock. To achieve on-time delivery which affects the customer's satisfaction, a specific time window $\left[a_{i}, b_{i}\right]$ is considered for each customer $i$. Therefore, we are penalized by $\mu$ or $\pi$ cost units for a time unit of earliness or tardiness, respectively. Fig. 1 illustrates the proposed problem.

The amount of GHG emissions released due to a liter of fuel consumption is denoted by GHG. Fuel consumption is different from a mountainous road to a flat road. So, we consider $\theta_{i j}$ as a road angel between two nodes $i$ and $j$.

A sustainable VRPCD which the social aspect is the freshness of delivered product $p$ to customer $i$ should be greater than the customer's expected freshness for product $p$, ex $x_{i p}$. Also, the maximum drivers' working hours $\max _{k}$ is minimized in order to improve their job satisfaction. In environmental aspect, GHG emissions are produced by vehicles should not exceed the allowed level, AL. Finally, the total cost, including inventory holding, transportation and penalty whether earliness or tardiness costs are considered in an economic viewpoint. 


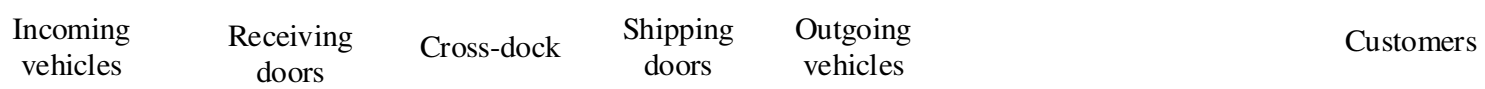

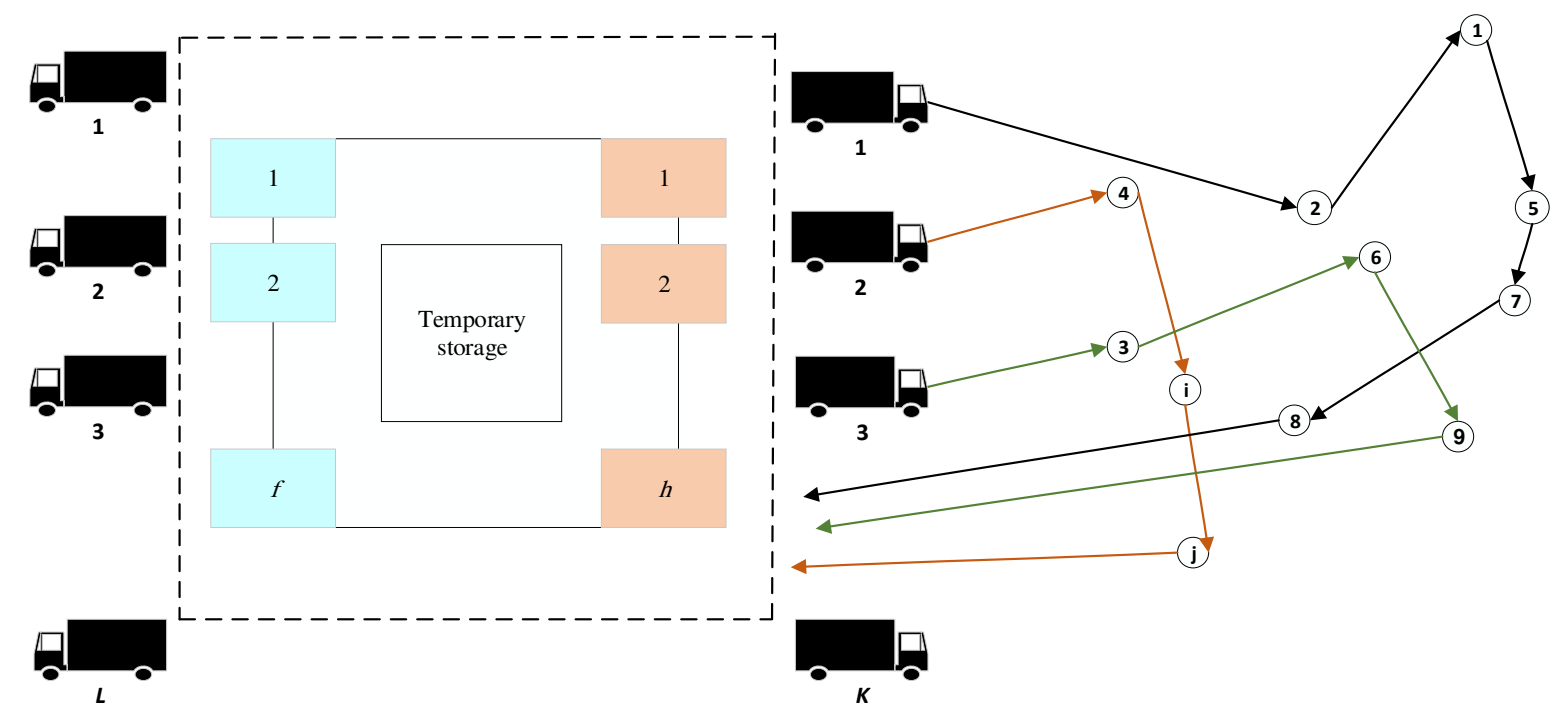

Fig. 1. The outline of the proposed problem

\subsection{Assumptions}

The following assumptions are made:

1. Temporary storage is unlimited

2. Single depot multi-vehicle problem with delivery time windows and no pickups is considered.

3. All outgoing vehicles are available at the zero time

4. $\quad$ Outgoing vehicles travel with constant speed from node $i$ to $j$

5. The total demands of customers do not exceed the total capacity of vehicles

6. The fleet is assumed heterogeneous, i.e., each vehicle has its own capacity, GHG emission, cost and average speed.

7. The products are fresh.

8. The freshness of products is starting to decrease when the products are unloaded at the cross-dock.

9. Drivers working hours are calculated when the cross-dock operations are started (at time zero).

10. Emission is function of air density, vehicle shape and gravity.

\subsection{Notations}

Set:

\begin{tabular}{ll}
\hline$N$ & $\begin{array}{l}\text { Set of all nodes including customers and cross dock, } i, j \in N=\{0,1, \ldots, n, n+1\}, \text { the cross dock is indicated by a } \\
\text { special node with index } 0 \text { or } n+1\end{array}$ \\
$F$ & Set of receiving doors, $f \in\{1, \ldots, F\}$ \\
$L$ & Set of incoming vehicles, $l \in\{0,1, \ldots, L, L+1\}$, where $l=0$ and $l=L+1$ define dummy incoming vehicles \\
$K$ & Set of outgoing vehicles, $k \in\{0,1, \ldots, K, K+1\}$, where $k=0$ and $k=K+1$ define dummy incoming vehicles \\
$H$ & Set of shipping doors, $h \in\{1, \ldots, H\}$ \\
$P$ & Set of product type, $p \in\{1, \ldots, P\}$ \\
\hline
\end{tabular}




\section{Parameters:}

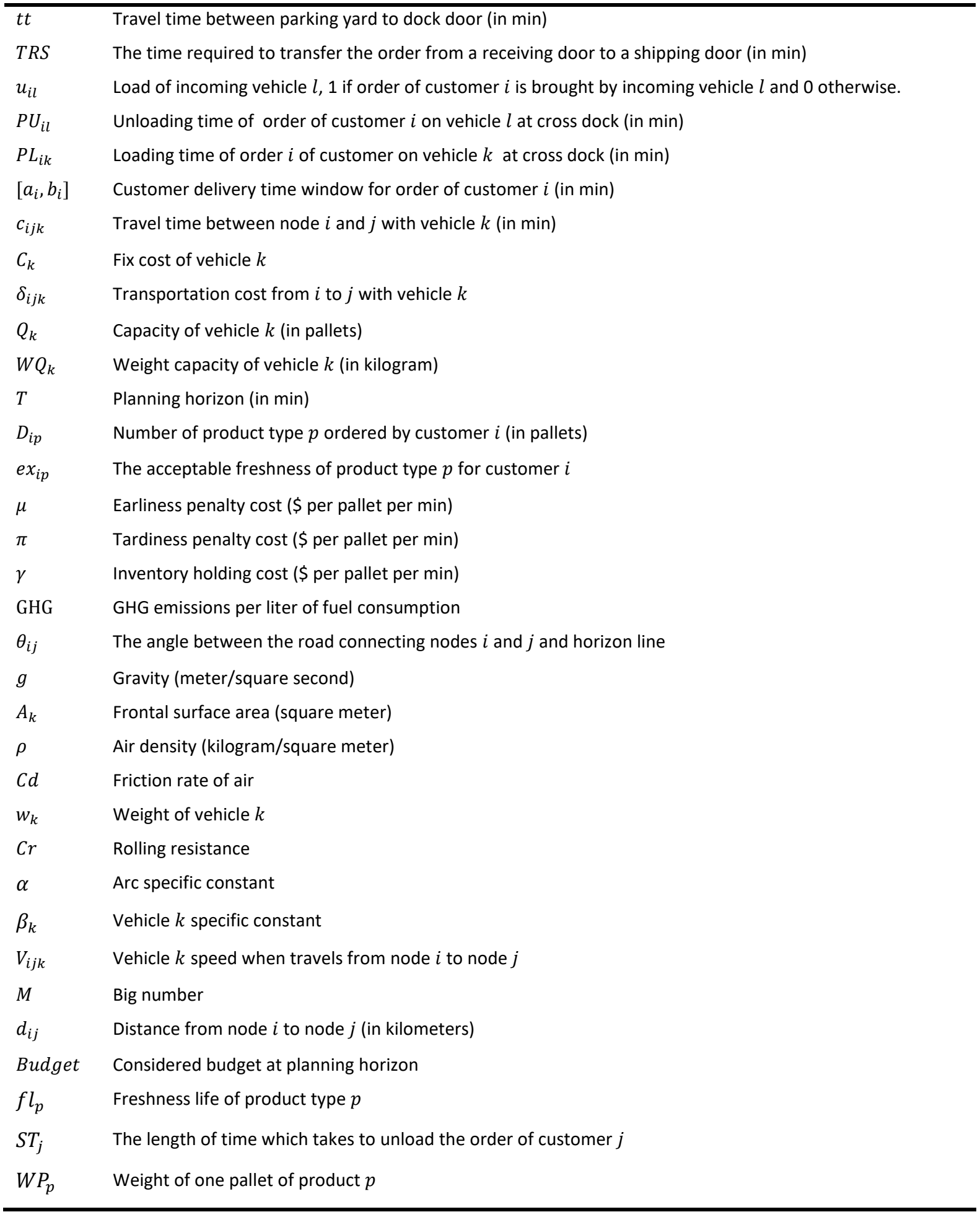


Variables:

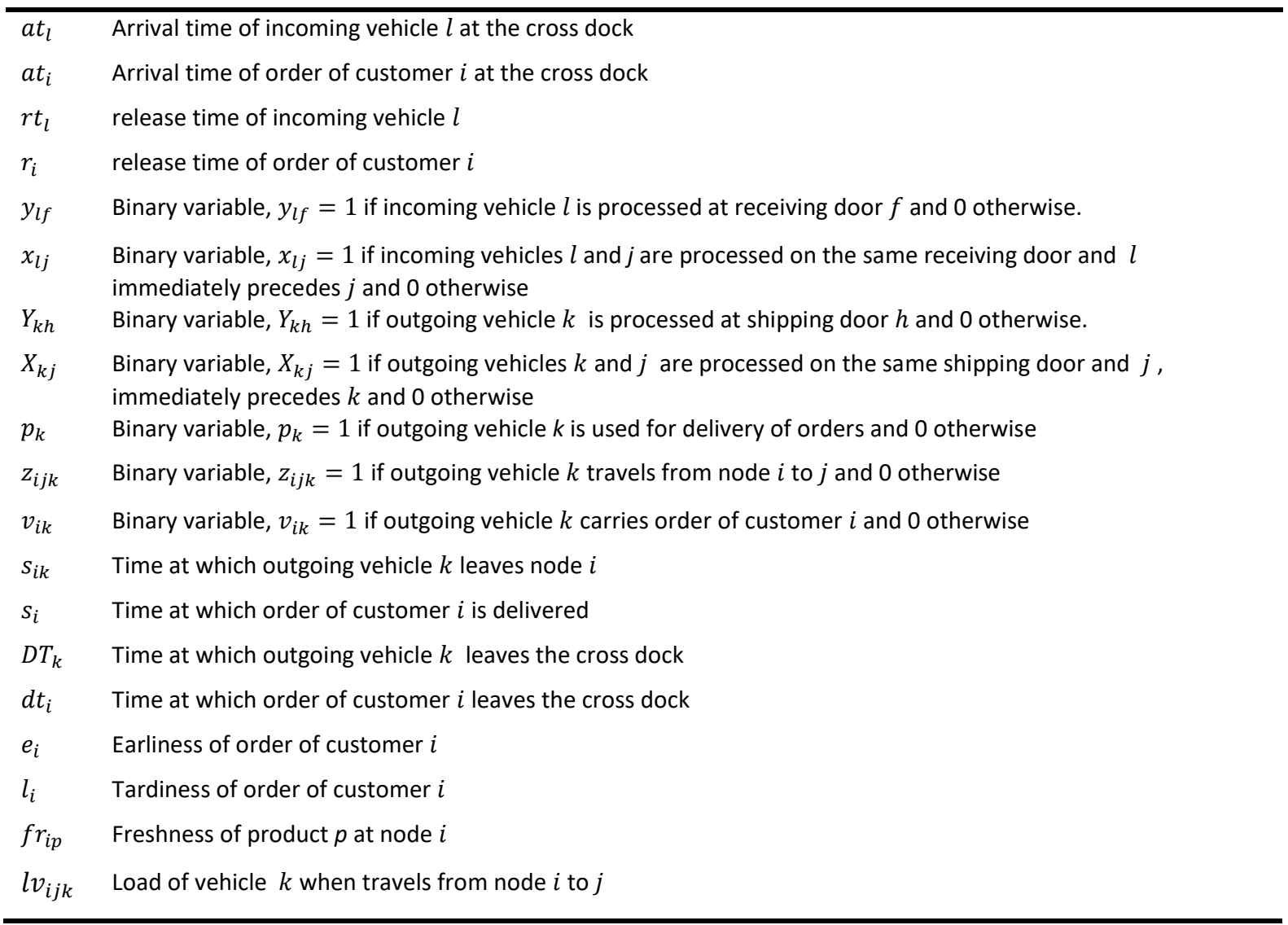

\subsection{Dimensions of Sustainability}

Three dimensions of sustainability (i.e., Economic, environmental and social aspects) are considered. In the economic viewpoint, different costs of cross-docking and delivery are taken into account. Indeed, penalty costs, including earliness and tardiness, inventory holding at cross-dock, vehicles fixed costs, transportation cost are considered, respectively. Therefore, Constraint (1) ensures that the total costs should not exceed a predetermined budget.

$$
\begin{gathered}
\text { Budget } \geq \sum_{i=1}^{n} \mu d_{i} e_{i}+\sum_{i=1}^{n} \pi d_{i} l_{i}+\sum_{i=1}^{n} \gamma\left(d t_{i}-r t_{i}\right) \sum_{p \in P} D+\sum_{i p} p_{k} C_{k} \\
+\sum_{i=0}^{n} \sum_{j=1, i \neq j}^{n+1} \sum_{k=1}^{K} \delta_{i j k}\left(z_{i j k} \times c_{i j k}\right)
\end{gathered}
$$

The environmental dimension is measured by the GHG (Green House Gas like CO2, CH4, and HFCs) emissions associated with the outgoing vehicles between cross-dock and customers. We employ the same approach as in Bektaş and Laporte (2011) for estimating fuel consumption and emissions. According to this model, the total amount of GHG from node $i$ to $j$ is measured as equation (2) which should be less than allowed emissions. The limit values of the input parameter variables AL which are characterized as the greatest and highest allowed values of the given parameters were defined on the basis of recommendations by the WHO. 
$G H G \sum_{i=0}^{n} \sum_{j=1}^{n+1} \alpha d_{i j} \sum_{k=1}^{K}\left(\left(w_{k} z_{i j k}\right)+l v_{i j k}\right)+\sum_{i=0}^{n} \sum_{j=1}^{n+1} \beta_{k} d_{i j} \sum_{k=1}^{K} V_{i j k}^{2} z_{i j k} \leq A L$

where $\alpha=g \sin \theta_{i j}+g * C r * \cos \theta_{i j}$ is an arc specific constant and $\beta=0.5 * C d * A_{k} * \rho$ is a vehicle specific constant. The first component measures the cost incurred by the load carried on the vehicle and the second component measures the cost implied by the speed of vehicle $k$.

The social dimension in this study included two important issues. The first social issue is measured through job satisfaction for drivers by minimization of the maximum working hours among all drivers in the planning horizon. This objective balances work hours and creates equity among the drivers to achieve fair working schedule. So overloaded drivers will work less than before and under-loaded drivers will fulfill this gap. The social objective is formulated as equation (3):

$\operatorname{Min} \operatorname{dmax}_{k}$

$d \max \geq s_{n+1, k}$

$\forall k \in\{1,2, \ldots, K\}$

where dmax is maximum of drivers working hours.

The second social issue is the freshness of each product should be greater than the allowed amount of freshness for customers. Due to decreasing quality of products by the time from cross-dock to customers, this factor for determining customer satisfaction is considered. Each product has a limited freshness-life under particular circumstances. Fig. 2 illustrates the freshness of the product over time. The important assumption in freshness calculation is products are unloaded at cross-dock with the highest freshness. We employ the same approach in Rahbari et al. (2019). Since expected freshness for each customer is not zero, the authors ignored the function after $T^{\prime}$.

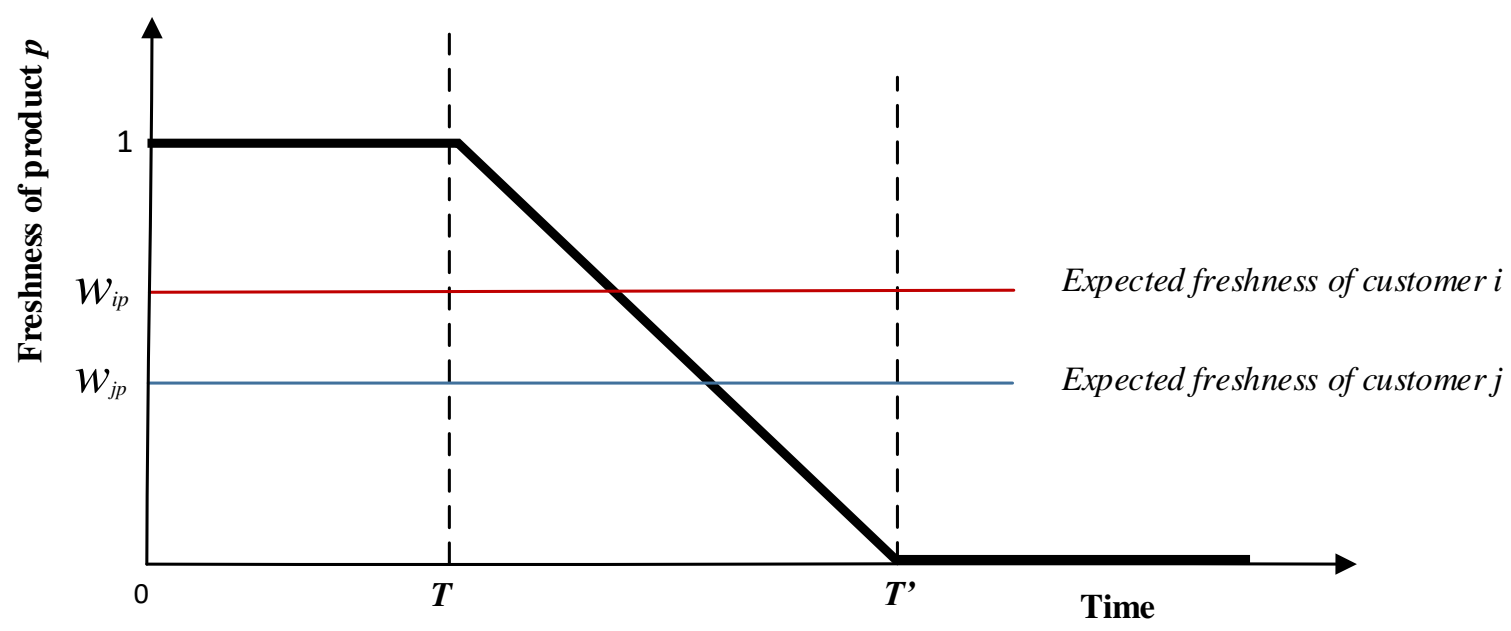

Fig. 2. The freshness of product over time

In Fig. 2, $t=0$ illustrates the time of product harvest, $T$ is the unloaded time at cross-dock and $T^{\prime}$ is the time which product lost acceptable freshness. According to Fig. 2 the freshness of the product formulation is: 


$$
\begin{array}{ll}
f r_{i p} \geq e x_{i p} & \forall i \in\{1,2, \ldots, n\} ; p \in\{1,2, \ldots, P\} ; D_{i p} \neq 0 \\
f r_{i p}=\frac{f l_{p}-\left(s_{i}-r_{i}\right)}{f l_{p}} & \forall i \in\{1,2, \ldots, n\} ; p \in\{1,2, \ldots, P\} ; D_{i p} \neq 0
\end{array}
$$

In this research, the freshness life of product after $T^{\prime}$ is ignored because the freshness must be greater than a positive threshold (the customer's expected freshness). Constraint (4) states the freshness of delivered any type of product for each customer should be greater than their expectation. Constraint (5) formulates the freshness function as a linear decreasing function. This function starts to decrease when orders released from cross dock. The other constraints are as follows:

$$
\begin{aligned}
& \sum_{k=1}^{K} \sum_{i=0, i \neq j}^{n} z_{i j k}=1 \\
& \forall J \in\{1,2, \ldots, n\} \\
& \sum_{j=1}^{n} z_{0 j k}=1 \\
& \forall k \in\{1,2, \ldots, K\} \\
& \sum_{i=1}^{n} z_{i, n+1, k}=1 \\
& \forall k \in\{1,2, \ldots, K\} \\
& \sum_{j=1}^{n} z_{j i k}-\sum_{j=1}^{n+1} z_{i j k}=0 \\
& \forall k \in\{1,2, \ldots, K\} ; i \in\{1,2, \ldots, n\} \\
& s_{i} \leq T \\
& D T_{j} \geq D T_{k}+\sum_{i=1}^{n} P L_{i j} v_{i j}-M\left(1-X_{k j}\right) \\
& \forall i \in\{1,2, \ldots, n\} \\
& \forall k, j \in\{1, \ldots, K\} ; k \neq j \\
& s_{i}-b_{i} \leq l_{i} \\
& \forall i \in\{1,2, \ldots, n\} \\
& a_{i}-s_{i} \geq e_{i} \\
& \forall i \in\{1,2, \ldots, n\} \\
& s_{j k} \geq s_{i k}+c_{i j k}+S T_{j}-M\left(1-z_{i j k}\right) \\
& \forall i \in\{0,1, \ldots, n\} ; \forall i \in\{1,2, \ldots, n+1\} ; i \\
& \neq j ; k \in\{1,2, \ldots, K\} \\
& s_{i} \geq s_{i k}-M\left(1-v_{i k}\right) \\
& \forall k \in\{1,2, \ldots, K\} ; i \in\{1,2, \ldots, n\} \\
& s_{i} \leq s_{i k}+M\left(1-v_{i k}\right) \\
& \forall k \in\{1,2, \ldots, K\} ; i \in\{1,2, \ldots, n\} \\
& \frac{1}{M} \sum_{i=0, i \neq j}^{n} z_{i j k} \leq v_{j k} \leq \sum_{i=0, i \neq j}^{n} z_{i j k} \\
& \forall k \in\{1,2, \ldots, K\} ; j \in\{1,2, \ldots, n\} \\
& \sum_{i=1}^{n} D_{i} v_{i k} \leq Q_{k} \\
& \forall k \in\{1,2, \ldots, K\} \\
& D T_{k} \geq r_{i}+P L_{i k}+T R S-M\left(1-v_{i k}\right) \\
& \forall k \in\{1,2, \ldots, K\} ; i \in\{1,2, \ldots, n\} \\
& d t_{i} \geq D T_{k}-M\left(1-v_{i k}\right) \\
& \forall k \in\{1,2, \ldots, K\} ; i \in\{1,2, \ldots, n\}
\end{aligned}
$$




$$
\begin{aligned}
& \begin{array}{l}
\sum_{h=1}^{H} Y_{k h}=1 \\
\sum_{f \in F} y_{l f}=1
\end{array} \\
& \forall k \in\{1,2, \ldots, K\} \\
& \forall l \in\{1,2, \ldots, L\} \\
& \forall l, j \in\{1,2, \ldots, L\} ; l \neq j ; \\
& \forall f \in\{1,2, \ldots, F\} \\
& \forall l \in\{1,2, \ldots, L\} ; i \in\{1,2, \ldots, n\} ; u_{i l}=1 \\
& r_{i} \geq r t_{l} \\
& r t_{l}=a t_{l}+t t+\sum_{i=1}^{n} P U_{i l} \\
& \forall l \in\{1,2, \ldots, L\} \\
& r t_{j} \geq r t_{l}+\sum_{i=1}^{n} P U_{i l}-M\left(1-x_{l j}\right) \\
& \sum_{j=0, j \neq i}^{n} f_{j i k}-\sum_{j=1, j \neq i}^{n+1} v l_{i j k}=\sum_{p=1}^{P} W P_{p} D_{i p} \quad \forall k \in\{1,2, \ldots, K\} ; i \in\{1,2, \ldots, n\} \\
& \left(\sum_{p \in P} W P_{p} D_{j p}\right) z_{i j k} \leq v l_{i j k} \leq\left(W Q_{k}-\sum_{p \in P} W P_{p} D_{i p}\right) z_{i j k} \quad \forall i \in\{0,1, \ldots, n\} ; \quad i \in\{1,2, \ldots, n+1\} ; i \neq j ; k \\
& \sum_{l=0, l \neq j}^{L} x_{l j}=1 \\
& \forall J \in\{1,2, \ldots, L\} \\
& \sum_{j=1, l \neq j}^{L+1} x_{l j}=1 \\
& x_{0 j}+x_{0 l}+y_{l f}+y_{j f} \leq 3 \\
& \left(X_{k j}-1\right) \leq Y_{k h}-Y_{j h} \leq\left(1-X_{k j}\right) \\
& \sum_{k=0, j \neq k}^{K} X_{k j}=1 \\
& \sum_{j=1, j \neq k}^{K+1} X_{k j}=1 \\
& X_{0 k}+X_{0 j}+Y_{k h}+Y_{j h} \leq 3 \\
& v_{i k} \leq p_{k} \\
& D T_{k}=s_{0 k} \\
& y_{l f} \in\{0,1\} \\
& x_{l j} \in\{0,1\} \\
& Y_{k h} \in\{0,1\} \\
& X_{k j} \in\{0,1\} \\
& z_{i j k} \in\{0,1\} \\
& v_{i k} \in\{0,1\} \\
& \forall l \in\{1,2, \ldots, L\} \\
& \forall l, j \in\{1,2, \ldots, L\} ; l \neq j ; f \in\{1,2, \ldots, F\} \\
& \forall k, j \in\{1,2, \ldots, K\} ; h \in\{1,2, \ldots, H\} ; k \neq j \\
& \forall j \in\{1,2, \ldots, K\} \\
& \forall k \in\{1,2, \ldots, K\} \\
& \forall k, j \in\{1,2, \ldots, K+1\} ; h \in\{1,2, \ldots, H\} ; k \\
& \neq j
\end{aligned}
$$

Constraint (6) states that every node is served once by a vehicle. Constraint (7) restricts each delivery route which should be started at the cross-dock. Constraint (8) ensures that every route should finish at the crossdock. Constraint (9) states the sequence of the outgoing vehicle's route of delivery. Constraint (10) guarantees each delivery order is completed within the planning horizon. Constraint (11) calculates departure time of 
vehicle $j$, if vehicle $j$ leaves cross-dock after $k$, immediately. Constraints (12) and (13) evaluate earliness and tardiness of customer $i$. Constraint (14) calculates leaving time of node $j$, if vehicle $k$ leaves node $j$ after node $i$, immediately. Constraints (15) and (16) imply delivery time for orders of each customer. Constraint (17) enforces $v_{j k}=1$, if vehicle $k$ carries order $j$. Constraint (18) ensures that the transported freight between two nodes should be less than the capacity of the vehicle. Constraint (19) implies that departure time of vehicle $k$ should be greater than release time of order $i$ if vehicle $k$ carries out order $i$. Constraint (20) ensures that departure time of order $i$ at cross-dock should be greater than departure time vehicle $k$ at cross-dock, if vehicle $k$ carries out order $i$. Constraint (21) implies that each outgoing vehicle should be processed on only one shipping door. Constraint (22) implies that an incoming vehicle should be processed at only one receiving door. Constraint (23) ensures that, if an incoming vehicle precedes another incoming vehicle, they should be at the same receiving door. Constraint (24) states incoming vehicle release time. Constraint (25) restricts the incoming truck release time that should be greater than arrival time. Constraint (26) ensures release time of incoming truck $j$ should be greater than arrival time of incoming vehicle $l$, if vehicle $j$ is processed after $l$, immediately. Constraints (27) and (28) restrict the arc flows. Constraint (29) ensures that each incoming vehicle has exactly one preceding incoming vehicle. Constraint (30) ensures that each incoming vehicle has exactly one succeeding receiving. Constraint (31) states that if both incoming vehicle $l$ and incoming vehicle $j$ immediately follow incoming vehicle 0 , they must be assigned to different receiving doors. Constraint (32) ensures that, if an outgoing vehicle precedes another outgoing vehicle, they should be at the same shipping door. Constraint (33) ensures that each outgoing vehicle has exactly one preceding outgoing vehicle. Constraint (34) ensures that each outgoing vehicle has exactly one succeeding shipping. Constraint (35) states that if both outgoing vehicle $k$ and outgoing vehicle $j$ immediately follow outgoing vehicle 0 , they must be on different outgoing doors. Constraint (36) ensures that if vehicle $k$ is used, related variable should be 1 . Constraint (37) states that departure time of vehicle $k$ at cross-dock is release time of vehicle $k$ at node 0 .

\section{Solution approach}

In this section, we proposed a matheuristic algorithm to solve the large-sized problems. Matheuristic as its name suggests is the hybridization of mathematical programming with metaheuristics. The proposed hybrid algorithm is based on the genetic algorithm (GA). The GA was introduced by Holland (1975) for the first time. A GA is a directed random search technique that evolves an initial population of solutions using genetic operators to create generations that are successively better with respect to the objective of interest and is especially suitable when the search space is unknown. In GA every individual or chromosome is encoded into a structure that represents the values of decision variables of the problem. The set of individuals forms the population. The chromosomes evolve through successive iterations, called generations. During each generation, the chromosomes are evaluated using some measures of fitness.

When the problem becomes more complicated, considering all the decisions in the chromosomes and preserving the feasibility of the solutions becomes very difficult. Therefore, some of the decisions can be made within a mathematical model (usually a mixed integer programming model). In the proposed approach, the 
decisions determined by each chromosome are transferred into an MIP model as the input (Sub-problem 1), where the other decisions are made by minimizing the objective function subject to some constraints. Then, the objective function value is returned to the GA to be used as the fitness value of that particular chromosome. Each of the individuals determines the values for the variables of the problem. Sub-problem 1 and the steps of the matheuristic are presented in Fig. 3.

Sub-problem 1:

Minimize $\operatorname{dmax}_{k}$

Subject to:

Constraints (2)-(5), (10)-(20), (24)-(28) and (36)-(37)

Step 1: Set the parameters

Step 2: Initialization:

Initialize the population.

Step 3: Evaluation:

For every individual, determine values of $y_{l f}, x_{l j}, X_{k j}, Y_{k h}, v_{i k}$ and $z_{i j k}$.

Using the above values as the parameters, solve Sub-problem 1 and return the optimal value of $d \max _{k}$ as the fitness of the individual.

Step 4: If the termination condition is satisfied go to step 7 otherwise go to step 5.

Step 5: Select the best solution among the entire population as parents, use cross-over and mutation operators to generate the new population as offspring.

Step 6: Go to step 3.

Step 7: Output the best solution.

Fig. 3. The matheuristic pseudo code

\subsection{Chromosome design}

In GA terminology, chromosome is an array that represents a candidate solution. In this case, chromosome is a $L+F+H+K+2 n-2$ array which is randomly generated. In the array, the assignment of each receiving/shipping vehicle to receiving/shipping doors and sequence of each vehicle is shown. This chromosome has four segments: the first segment is related to sequence and assignment of incoming vehicles. The integer numbers between 1 to $L+F-1$ are generated randomly. The second segment is related to customers which is generated randomly. The third segment is related to sequence and assignments of outgoing vehicles. The integer numbers between 1 to $K+H-1$ is generated randomly. The forth segment is related to priority of customers. 
For an example, consider a problem with four incoming vehicles $(L=4)$, two receiving dock-doors $(F=$ $2)$, five shipping vehicles ( $K=5)$, two shipping dock-doors $(H=2)$ and eight customers $(n=8)$. Fig. 4 illustrates the chromosome of this example. This chromosome tells us the sequence and assignment of receiving/shipping vehicles in receiving/ shipping dock-doors and the sequence of customers. The numbers that are greater than number of vehicles separate dock-doors from each other. The first position which its value is greater than four is third position. So vehicles number 4 and 1 are processed on receiving dock-door number 1 , respectively, i.e. $y_{41}=1, y_{11}=1, x_{04}=1, x_{41}=1$ and $x_{15}=1$. Vehicles number 2 and 3 are processed on receiving dock-door number 2 , respectively.

The second segment is related to customer's node. For third segment, the other sequence and assignment of outgoing vehicles are same as incoming vehicles procedure.

To decoded $v_{i k}$, the first three segments of chromosome are considered. The products are transferred using FCFS rule from incoming vehicle $l$ to outgoing vehicle $k$ based on the truck sequence. In this example, the products carried by incoming vehicle 4 are firstly allocated to outgoing vehicle 1 according to customer sequence, as required. The next outgoing vehicles in the shipping sequence $(3,4,2$ and 5 respectively) pick up the remaining products from incoming vehicle 4 if required, until all products are allocated. These processes are repeated with the next incoming vehicles $(1,2$, and 3 respectively) until all incoming products are allocated to outgoing vehicles. Incoming vehicle 4 brought orders 2, 3 and 8. Outgoing vehicle 1 picks up order 2 according to the order sequence. Outgoing vehicle 3 picks up order 3 , also order 8 is allocated to outgoing vehicle 3 i.e. $v_{21}=1, v_{33}=1$ and $v_{83}=1$. Fig. 5 demonstrates this example. 


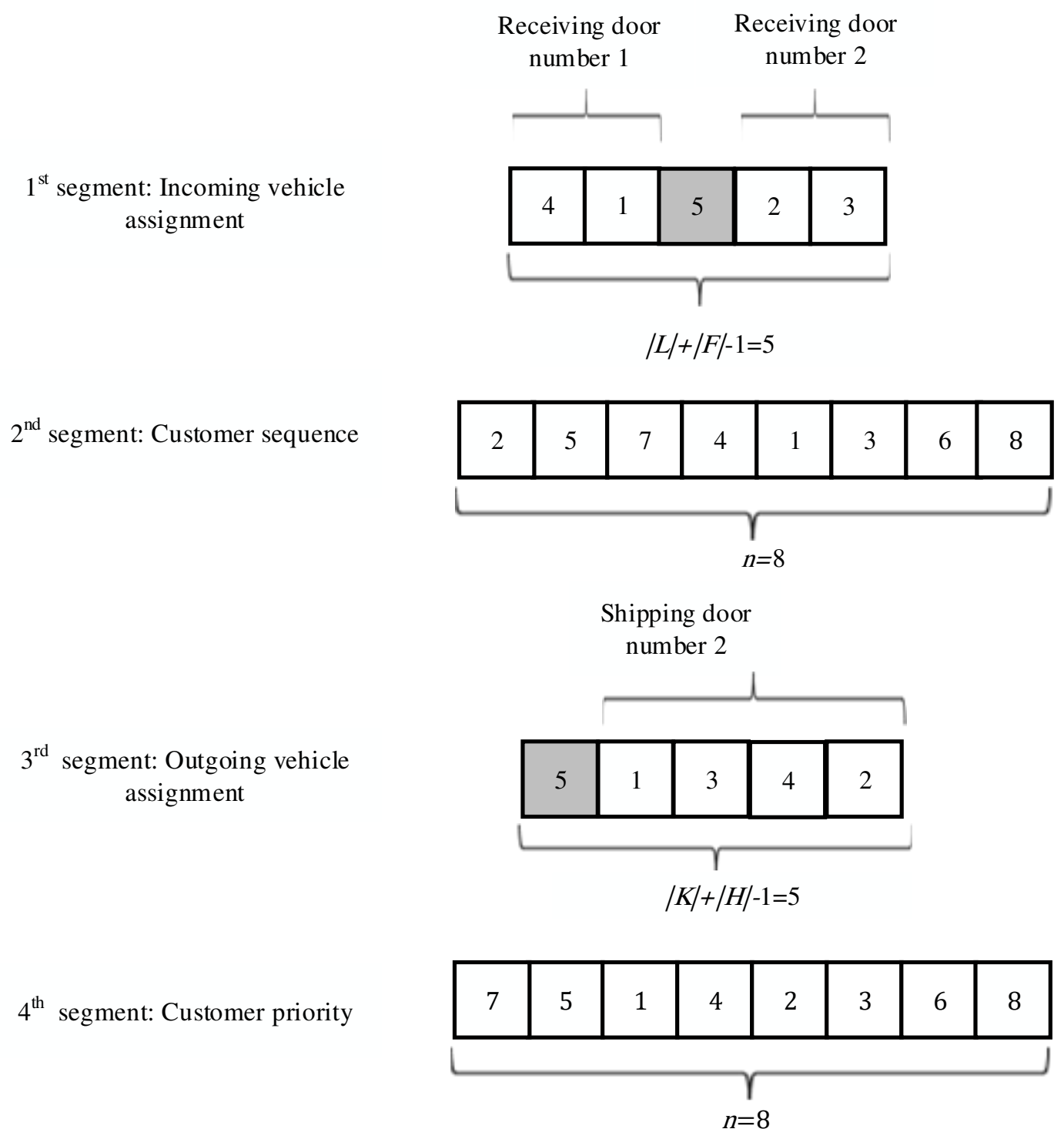

Fig. 4. Solution representation for an example 


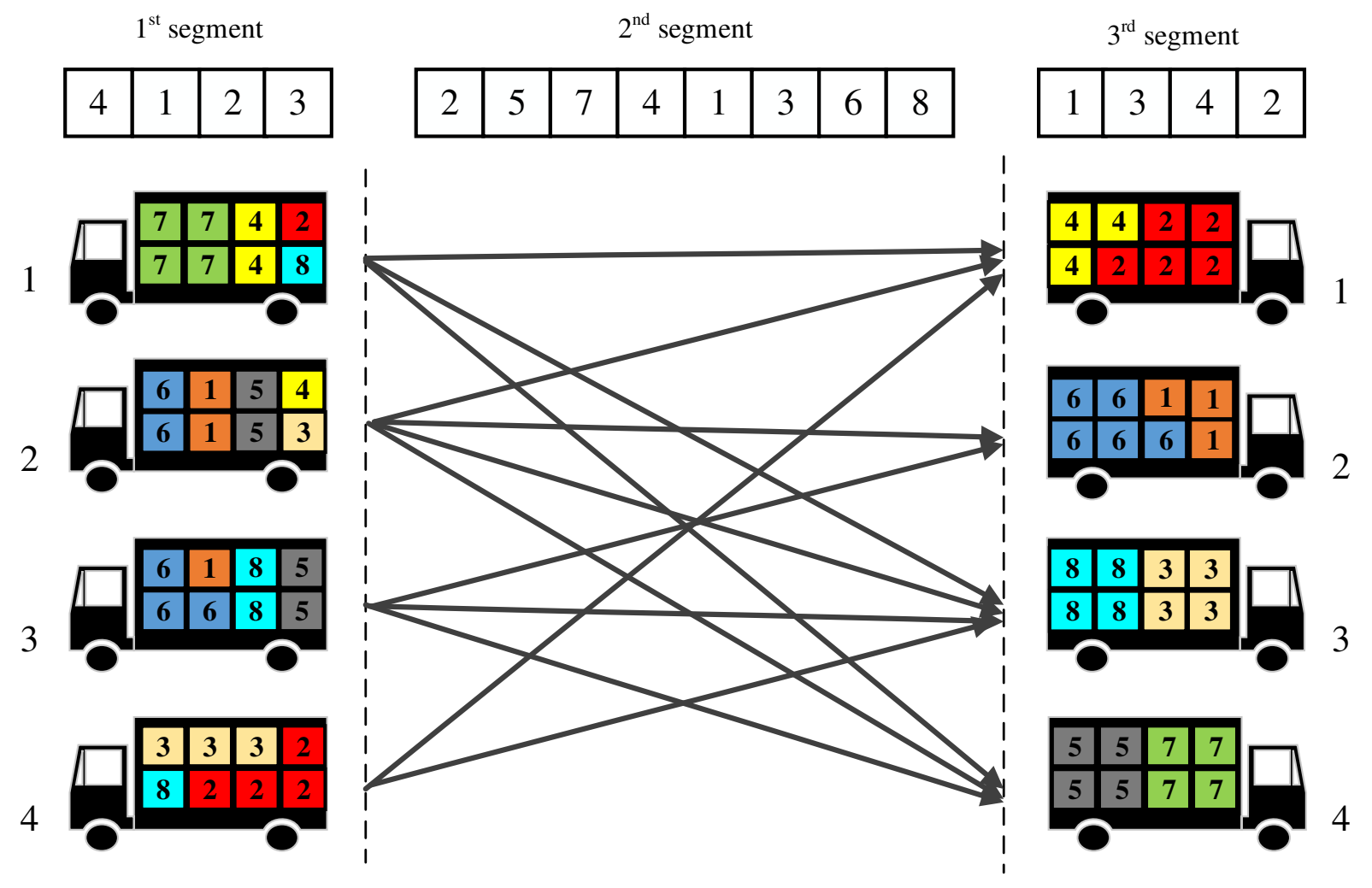

Fig. 5. An example of product transfer method using FCFS rule

To decode $z_{i j k}$, the fourth segment of the chromosome is considered. The products are assigned to the outgoing vehicle in previous step. Now, customer sequence for each truck should be determined. In this example, outgoing vehicle 1 carries orders of customers 4 and 2 . $\ln 4^{\text {th }}$ segment, priority of customer 4 is higher than customer 2 . So outgoing vehicle 1 first visits customer 4 . It should be noted that $4^{\text {th }}$ segment is used to generate different solutions.

\subsection{Evaluation}

In genetic algorithm for evaluation of random chromosome quality, a fitness function is considered. The fitness function consists of minimizing the maximum working hours for drivers.

\subsection{Selection procedure}

At this stage of the algorithm, the well-known standard roulette wheel selection method is applied for selection. A number of chromosomes are selected from the initial population in order to start the crossover stage.

\subsection{Crossover}

Crossover operator is used to provide variant chromosomes and cover the solution space more thoroughly. The implemented crossover is a variant of the standard one-point crossover operator and is described in Fig. 6 . 
Given the assumed probability of intersection (crossover), we determine semi-randomly the genes that will be affected from each parent chromosome:

First, generate a random number between 1 to 4 , according to segments of the chromosome. Second, according to random number, apply the cross-over operator. For instance, for random number 1 , cross-over is applied in first segment of chromosome, other segments will be unchanged. Fig. 6 illustrates proposed crossover performance. The genes (trucks) from parent 1 before cross-over point, will be copied on child 1, remaining genes of child 1 for first segment are affected by parent 2. For child 2, the genes before cross-over point will be copied from parent 2. Parent 1 will affect the remaining genes of child 2 . We replace the children with parents if they optimize the fitness of the parents. The others will be the same.

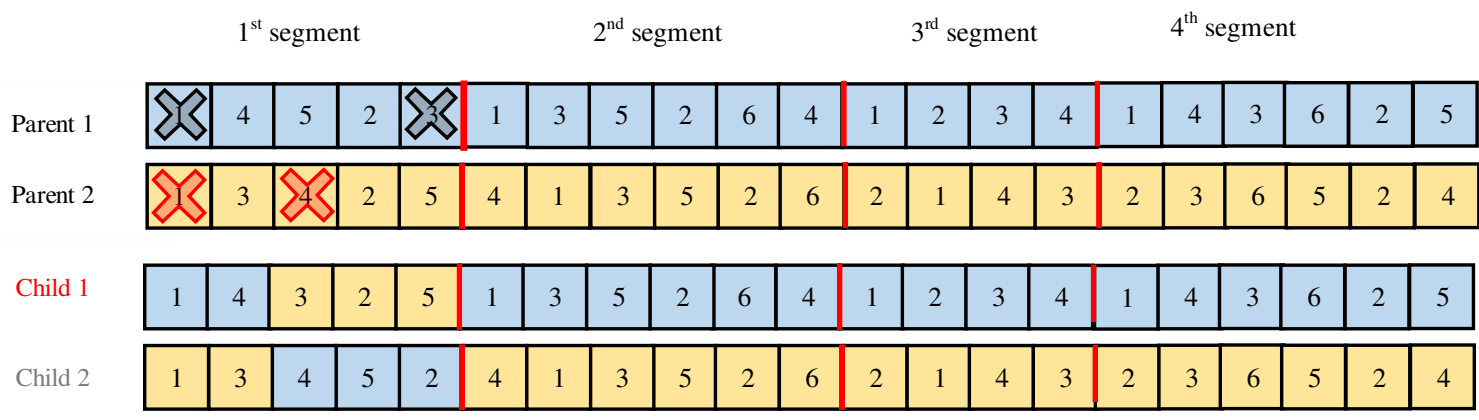

Fig. 6. Single-point crossover

\subsection{Mutation}

The implemented Mutation is used to replace a gene with another gene in the same chromosome. The probability of gene selection is the same. As previously mentioned, a random number is generated. If the random number is 2 , the mutation consists of selecting a subset of two genes from the node string of the chromosome and swapping them. Fig. 7 provides a schematic view of the proposed mutation operator using a numerical example.

\begin{tabular}{|c|c|c|c|c|c|c|c|c|c|c|c|c|c|c|c|c|c|c|c|c|}
\hline \multicolumn{5}{|c|}{$1^{\text {st }}$ segment } & \multicolumn{6}{|c|}{$2^{\text {nd }}$ segment } & \multicolumn{4}{|c|}{$3^{\text {rd }}$ segment } & \multicolumn{6}{|c|}{$4^{\text {th }}$ segment } \\
\hline 4 & 1 & 5 & 2 & 3 & 1 & 3 & 5 & 2 & 1 & 6 & 4 & 1 & 3 & 2 & 1 & 4 & 3 & 6 & 2 & 5 \\
\hline 1 & 4 & 5 & 2 & 3 & 1 & 3 & 6 & 2 & 1 & 5 & 4 & 1 & 3 & 2 & 1 & 4 & 3 & 6 & 2 & 5 \\
\hline
\end{tabular}

Fig. 7. Mutation operator

\subsection{Termination criteria}


In this paper, the modified GA terminates when the number of function evaluations reaches to the maximum value (Max NFE). This method is a combination of mathematical programming and metaheuristic, so a smaller NFE can be reached in a reasonable time.

\section{Computational results}

In this section, numerical results on the performance of the proposed Matheuristic is presented. The algorithm has been coded in the MATLAB and GAMS software. First, parameter setting and test problems are discussed. Then, a real case study is provided to demonstrate the applicability of the model in real-world applications.

\subsection{Parameter setting}

Only searching and solution representation procedures do not affect the performance of the proposed algorithms, also parameter setting is important for finding well solution and convergence. To determine the appropriate values of the GA parameters Taguchi method is applied. The preliminary experiments are conducted with three different values of each parameter. The parameters are crossover rate, mutation rate and population size. For small and large-sized problems, three levels of parameters are proposed. The levels of these parameters are shown in Table 3. The parameters of GA, i.e. crossover rate, mutation rate and population size are set to 0.3 , 0.5 and 30 for problems.

Table 3. Selected levels of parameters in Taguchi experiment.

\begin{tabular}{cccc}
\hline \multirow{2}{*}{ Levels } & \multicolumn{3}{c}{ Parameters } \\
\cline { 2 - 4 } & Population size & Crossover rate & Mutation rate \\
\hline 1 & 15 & 0.1 & 0.3 \\
2 & 30 & 0.3 & 0.5 \\
0.5 & 0.5 & 0.7 \\
\hline
\end{tabular}

\subsection{Test problems}

To validate the proposed mathematical formulation and meta-heuristic algorithm, computational experiments are designed with various operating situations. The test problems are categorized into two groups: 1) small problems 1-10 and 2) large problems 11-20. Small-size demonstrations are provided to extrapolate important insights from the derived results while large-size implementations are presented to examine the performance of the proposed algorithm. Furthermore, a case study is applied to know the performance of the solution. We should note that the small-sized problems are subset of the case study.

Our objective is to obtain the solution quality rather than solution speed. Using the mathematical programming in the solution method, leads to a slower fitness evaluation while at the same time, the mathematical programming prevents generation of infeasible solutions and does not require the repair process or penalty methods. In small-sized problems, to validate the solution method, we first calculate the value of 
objective function for the specific NFE (300) in GA. Then CPLEX solver is executed to obtain a solution around the value of the objective function obtained from GA. Then the value of relative error $(R E)$ is calculated according to equation (38):

$$
R E=\frac{O \cdot F \cdot-X}{X} \times 100
$$

$O . F$. is the best value of the objective function found by the algorithm.

$X$ is the optimal value of the objective function (lower bound) in CPLEX in small size (large size) problems.

The small amount of RE indicates the efficiency of the proposed algorithm in small dimensions due to the lower execution time than the execution time of CPLEX.

To validate on large scale, we run CPLEX for 3600 seconds and then run GA with NFE $=500$. The results show that for some cases CPLEX has not been able to find an answer during this time, and for some that have been answered, the result of the MGA algorithm has better quality. In addition, the lower bound of CPLEX has been used to calculate RE.

In this section, 20 problems are randomly-generated and solved. The obtained results are presented. Tables 4 and 5 show the results for the small-sized and large-sized problem instances, respectively.

Table 4. The objective function values and CPU times in small-sized problems

\begin{tabular}{|c|c|c|c|c|c|c|c|c|c|}
\hline \multirow{3}{*}{ Instance } & \multicolumn{8}{|c|}{ Solution of models } & \multirow{3}{*}{ RE } \\
\hline & & & & & \multicolumn{2}{|c|}{ CPU time (s) } & \multicolumn{2}{|c|}{ O.F. } & \\
\hline & $l$ & $k$ & $p$ & $n$ & CPLEX & MGA & CPLEX & MGA & \\
\hline 1 & 3 & 5 & 3 & 8 & 272 & 204 & 214.2 & 214.2 & 0 \\
\hline 2 & 4 & 4 & 4 & 9 & 298 & 116 & 238.1 & 238.1 & 0 \\
\hline 3 & 4 & 5 & 5 & 8 & 334 & 244 & 223.4 & 223.9 & $0.2 \%$ \\
\hline 4 & 3 & 3 & 4 & 9 & 251 & 101 & 243.2 & 243.7 & $0.2 \%$ \\
\hline 5 & 3 & 5 & 4 & 10 & 521 & 187 & 232.3 & 232.3 & 0 \\
\hline 6 & 3 & 4 & 5 & 8 & 241 & 103 & 225.4 & 225.4 & 0 \\
\hline 7 & 4 & 5 & 3 & 9 & 463 & 350 & 230.1 & 230.1 & 0 \\
\hline 8 & 3 & 4 & 5 & 9 & 301 & 211 & 232.4 & 232.7 & $0.1 \%$ \\
\hline 9 & 4 & 4 & 3 & 10 & 479 & 202 & 241.8 & 241.8 & 0 \\
\hline 10 & 4 & 5 & 5 & 10 & 875 & 228 & 236.4 & 236.8 & $0.1 \%$ \\
\hline Average & & & & & & & & & $0.06 \%$ \\
\hline
\end{tabular}

Table 5. The objective function values and CPU times in large-sized problems

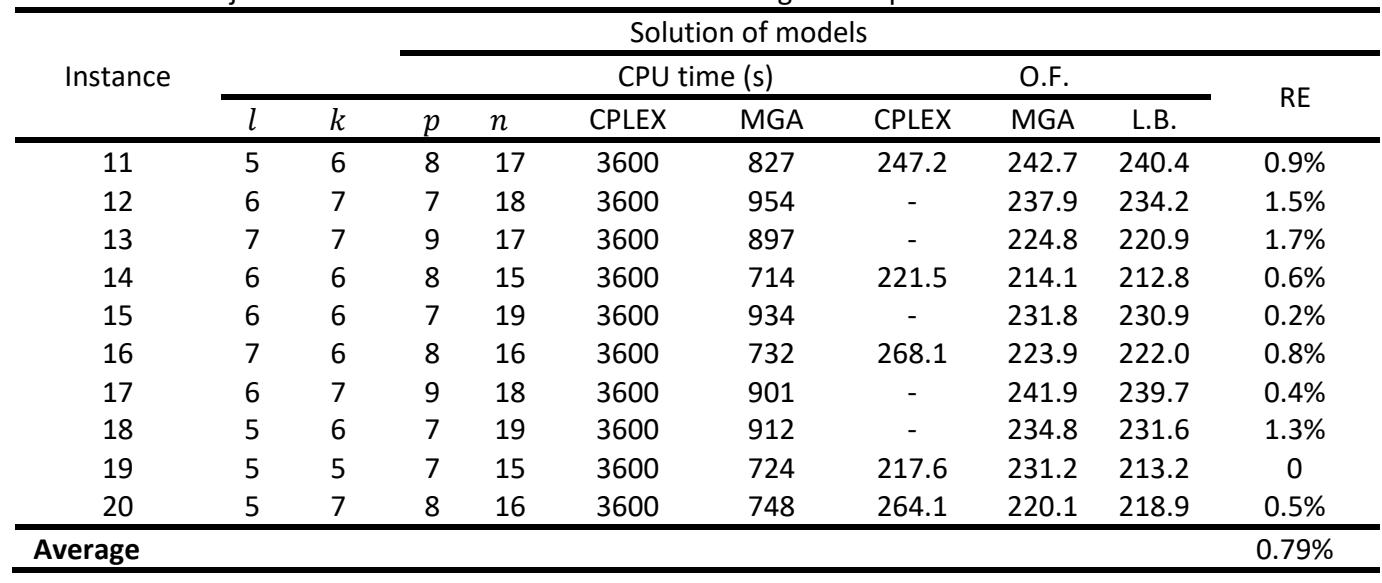




\subsection{Case study}

To investigate the applicability of the proposed method in real-world applications, a real case study is presented. This case shows how the model was applied on the routing of vehicles in Tehran. The underlying transportation network includes a cross-dock which is located in Shahre-Qods and 20 customers. A 20 years old company with a cross dock and some farms around the town is considered. Some vehicles are devoted to transfer vegetables from different farms to cross dock. In the cross dock, five type of vegetables are combined together according to customer's orders. Each product has a descending freshness. At the early stages, the products keep their freshness at the high level until arriving to cross dock. As products are fresh and time windows is considered, the presence of sorting and combination system to accelerate delivery time is unavoidable. Six heterogeneous vehicles are ready at the starting time to carry the orders to Tehran city. The customers are located in the city center of Tehran. Fig. 8. illustrates the location of the cross-dock and the customers in the city. It should be noted that Tehran is a large city, and the distances between black circles are at least several kilometers.

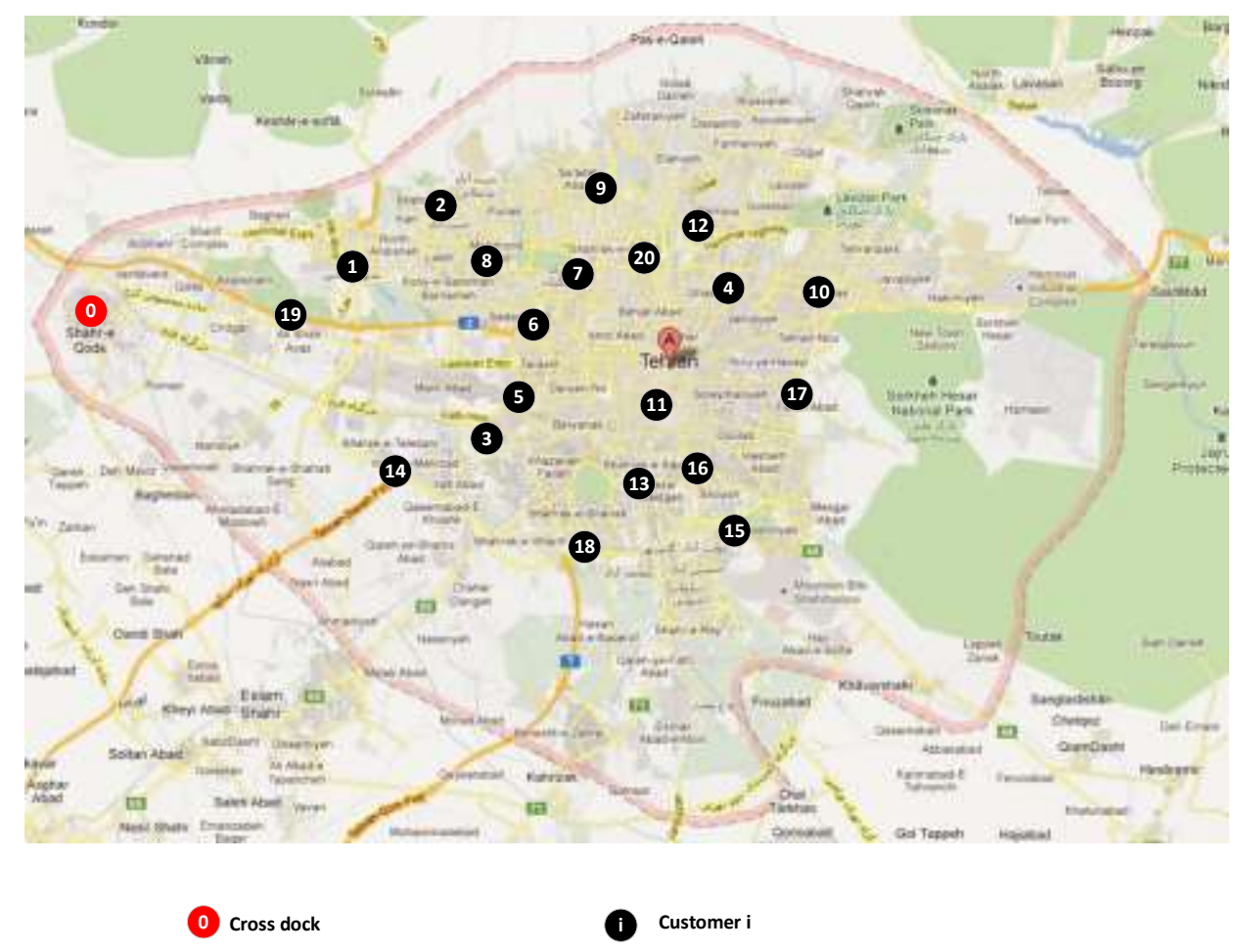

Fig. 8. The dispersion of the customer nodes. 
Five perishable products should be transferred to customer nodes by three different types of vehicles to satisfy customers demands. The freshness of products decreases with time. Travel times (illustrated in Table 6) are provided by using Google Maps. The cross-dock operations are started at 6:30 AM.

Table 6. Travel time between two nodes via google map

\begin{tabular}{|c|c|c|c|c|c|c|c|c|c|c|c|c|c|c|c|c|c|c|c|c|c|}
\hline & 1 & 2 & 3 & 4 & 5 & 6 & 7 & 8 & 9 & 10 & 11 & 12 & 13 & 14 & 15 & 16 & 17 & 18 & 19 & 20 & 21 \\
\hline 0 & 44 & 60 & 62 & 80 & 65 & 78 & 82 & 63 & 65 & 90 & 70 & 95 & 75 & 40 & 75 & 78 & 80 & 48 & 40 & 86 & 0 \\
\hline 1 & 0 & 6 & 10 & 20 & 8 & 10 & 15 & 8 & 10 & 30 & 20 & 20 & 24 & 12 & 39 & 25 & 43 & 21 & 6 & 23 & 38 \\
\hline 2 & 6 & 0 & 25 & 26 & 20 & 17 & 18 & 12 & 8 & 26 & 22 & 20 & 33 & 26 & 40 & 30 & 36 & 32 & 15 & 16 & 44 \\
\hline 3 & 11 & 28 & 0 & 15 & 8 & 12 & 19 & 17 & 24 & 26 & 10 & 30 & 13 & 12 & 19 & 17 & 24 & 11 & 16 & 24 & 40 \\
\hline 4 & 19 & 28 & 18 & 0 & 15 & 13 & 10 & 18 & 21 & 12 & 8 & 15 & 16 & 31 & 21 & 8 & 14 & 21 & 36 & 6 & 50 \\
\hline 5 & 8 & 24 & 10 & 18 & 0 & 8 & 12 & 13 & 19 & 16 & 10 & 24 & 12 & 17 & 24 & 20 & 25 & 14 & 17 & 20 & 42 \\
\hline 6 & 16 & 16 & 10 & 12 & 8 & 0 & 8 & 9 & 14 & 23 & 12 & 25 & 20 & 28 & 32 & 16 & 28 & 24 & 20 & 16 & 47 \\
\hline 7 & 18 & 16 & 23 & 14 & 13 & 10 & 0 & 10 & 15 & 23 & 16 & 16 & 24 & 36 & 31 & 16 & 26 & 30 & 36 & 9 & 50 \\
\hline 8 & 12 & 15 & 20 & 20 & 15 & 12 & 11 & 0 & 11 & 32 & 20 & 29 & 26 & 30 & 36 & 23 & 30 & 30 & 21 & 19 & 44 \\
\hline 9 & 12 & 10 & 21 & 20 & 20 & 16 & 16 & 14 & 0 & 25 & 26 & 15 & 32 & 36 & 36 & 26 & 33 & 34 & 27 & 11 & 49 \\
\hline 10 & 32 & 30 & 30 & 12 & 17 & 28 & 23 & 35 & 25 & 0 & 19 & 11 & 20 & 36 & 17 & 11 & 9 & 24 & 41 & 13 & 69 \\
\hline 11 & 20 & 23 & 12 & 8 & 10 & 10 & 18 & 20 & 24 & 20 & 0 & 22 & 9 & 24 & 19 & 9 & 17 & 13 & 29 & 15 & 45 \\
\hline 12 & 28 & 24 & 34 & 18 & 22 & 29 & 19 & 26 & 15 & 12 & 22 & 0 & 28 & 43 & 21 & 17 & 19 & 26 & 42 & 9 & 70 \\
\hline 13 & 28 & 36 & 13 & 20 & 14 & 20 & 21 & 26 & 32 & 22 & 10 & 28 & 0 & 24 & 16 & 11 & 14 & 10 & 31 & 21 & 43 \\
\hline 14 & 10 & 22 & 15 & 35 & 15 & 30 & 32 & 32 & 36 & 34 & 22 & 43 & 24 & 0 & 31 & 29 & 36 & 14 & 13 & 41 & 36 \\
\hline 15 & 48 & 40 & 21 & 24 & 23 & 35 & 36 & 34 & 38 & 17 & 20 & 20 & 17 & 32 & 0 & 12 & 9 & 19 & 34 & 30 & 56 \\
\hline 16 & 20 & 30 & 17 & 8 & 20 & 18 & 17 & 23 & 27 & 10 & 10 & 19 & 10 & 30 & 14 & 0 & 13 & 19 & 31 & 20 & 47 \\
\hline 17 & 50 & 35 & 21 & 11 & 26 & 25 & 28 & 30 & 33 & 10 & 18 & 20 & 14 & 37 & 10 & 14 & 0 & 28 & 41 & 20 & 60 \\
\hline 18 & 26 & 30 & 11 & 20 & 13 & 26 & 30 & 29 & 35 & 24 & 13 & 27 & 10 & 14 & 20 & 20 & 28 & 0 & 30 & 29 & 40 \\
\hline 19 & 9 & 18 & 20 & 40 & 18 & 20 & 34 & 21 & 26 & 40 & 27 & 40 & 32 & 13 & 32 & 32 & 41 & 31 & 0 & 34 & 35 \\
\hline 20 & 27 & 19 & 24 & 8 & 22 & 20 & 9 & 20 & 11 & 15 & 15 & 10 & 21 & 41 & 30 & 21 & 20 & 32 & 37 & 0 & 53 \\
\hline
\end{tabular}

There are six incoming vehicles and six outgoing vehicles as the transportation system. In addition, there are three receiving dock doors for unloading purposes, and three shipping dock doors for loading the consolidated shipments into the outgoing vehicles. The other parameters are given in Table 7. In addition, the parameter values related to the carbon dioxide emissions are extracted from Bektaş and Laporte (2011).

Table 7. Parameters of the case study

\begin{tabular}{|c|c|c|c|}
\hline $\mathrm{PL}_{i p}=D_{i p}$ & $f l_{p}=\left[\begin{array}{llllll}220 & 250 & 300 & 300 & 500\end{array}\right]$ & $\pi=0.3$ & $\mu=0.1$ \\
\hline$\delta_{i j k}=0.1$ & $w_{k}=\left[\begin{array}{llllll}5 & 6 & 7 & 5 & 6 & 7\end{array}\right]^{*} 1000$ & $a_{i}=U[20,160]$ & $P U=15$ \\
\hline Budget $=2000$ & $Q_{k}=\left[\begin{array}{llllll}20 & 22 & 25 & 20 & 22 & 25\end{array}\right]$ & $T=1440$ & $b_{i}=a_{i}+U[20,40]$ \\
\hline$\gamma=0.4$ & $C_{k}=\left[\begin{array}{llllll}20 & 30 & 40 & 20 & 30 & 40\end{array}\right]$ & $t t=5$ & $S T_{j}=\sum_{p} D_{j p}$ \\
\hline
\end{tabular}

The problem's objective is to find optimum decisions about sequencing, scheduling and routing of vehicles to minimize the maximum working hours of drivers (load leveling of working hours). Also, time windows are considered to limit the acceptable arrival time and determine penalty costs for earliness and tardiness service. Considering the freshness and time windows together may seem redundant, but the freshness life has a different function and tries to make the model select shorter routes.

Since, our algorithm is fix and optimize algorithm, we applied our model in MATLAB and CPLEX (generating variables in MATLAB and optimize them in CPLEX). Once we implemented the algorithm to the case study with 
fix and optimize approach. We have some binary variables which is generated in MATLAB except $z_{i j k}$. As this decision should be made at CPLEX, so we can have optimization in CPLEX. We should note $z_{i j k}$ represents sequence of customers for each vehicle. The forth part of chromosome in fig 4. Belongs to $z_{i j k}$ generation. So we implemented MGA to the case study without forth segment of chromosome. This algorithm is without the order of the customers in the chromosome (fourth part) and considering it in CPLEX software in order to optimize it is mentioned MGA. Furthermore, we applied algorithm with considering $z_{i j k}$ at chromosome at equal times (GA).

Then the convergence diagrams of this algorithm are illustrated for both GA and MGA in Fig. 9. It is observed in long term decision when the order of the customers is optimized in CPLEX, better results are obtained compared to the case that all the decisions are made in GA. Also, Table 8 illustrates the routing and scheduling of vehicles at the cross-dock. Furthermore, the routing of vehicle is displayed in Fig. 10.

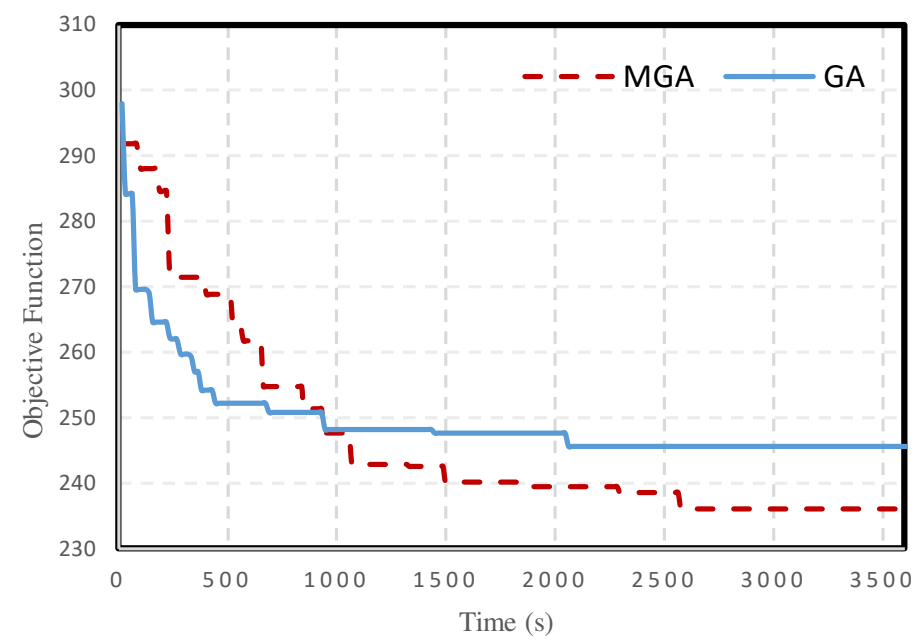

Fig. 9. The convergence of the proposed GA and MGA for the case study. 
Table 8. The schedule resulted from optimizing $O F(O F=236$, total $\operatorname{cost}=472)$

\begin{tabular}{|c|c|c|c|c|c|}
\hline Receiving Door & $\begin{array}{l}\text { Sequence (schedule) of } \\
\text { incoming vehicles }\end{array}$ & Shipping door & $\begin{array}{c}\text { Sequence (schedule) of } \\
\text { outgoing vehicles }\end{array}$ & outgoing vehicle & Visited nodes (schedule) \\
\hline 1 & $3(0) \rightarrow 1(20)$ & 1 & $2(35) \rightarrow 1(69)$ & 1 & $0(80) \rightarrow 18(132) \rightarrow 3(150) \rightarrow 11(161) \rightarrow 4(174) \rightarrow 21(228)$ \\
\hline 2 & $4(0) \rightarrow 2(20)$ & 2 & $4(45)$ & 2 & $0(65) \rightarrow 19(105) \rightarrow 20(139) \rightarrow 10(154) \rightarrow 12(165) \rightarrow 9(183) \rightarrow 21(236)$ \\
\hline \multirow[t]{4}{*}{3} & $5(0) \rightarrow 6(20)$ & 3 & $3(20) \rightarrow 5(67)$ & 3 & $0(65) \rightarrow 1(121) \rightarrow 8(136) \rightarrow 7(154) \rightarrow 6(171)) \rightarrow 5(186) \rightarrow 21(233)$ \\
\hline & & & & 4 & $0(65) \rightarrow 15(147) \rightarrow 17(157) \rightarrow 16(172) \rightarrow 13(183) \rightarrow 21(230)$ \\
\hline & & & & 5 & $0(80) \rightarrow 14(120) \rightarrow 2(147) \rightarrow 21(198)$ \\
\hline & & & & 6 & Not used \\
\hline
\end{tabular}

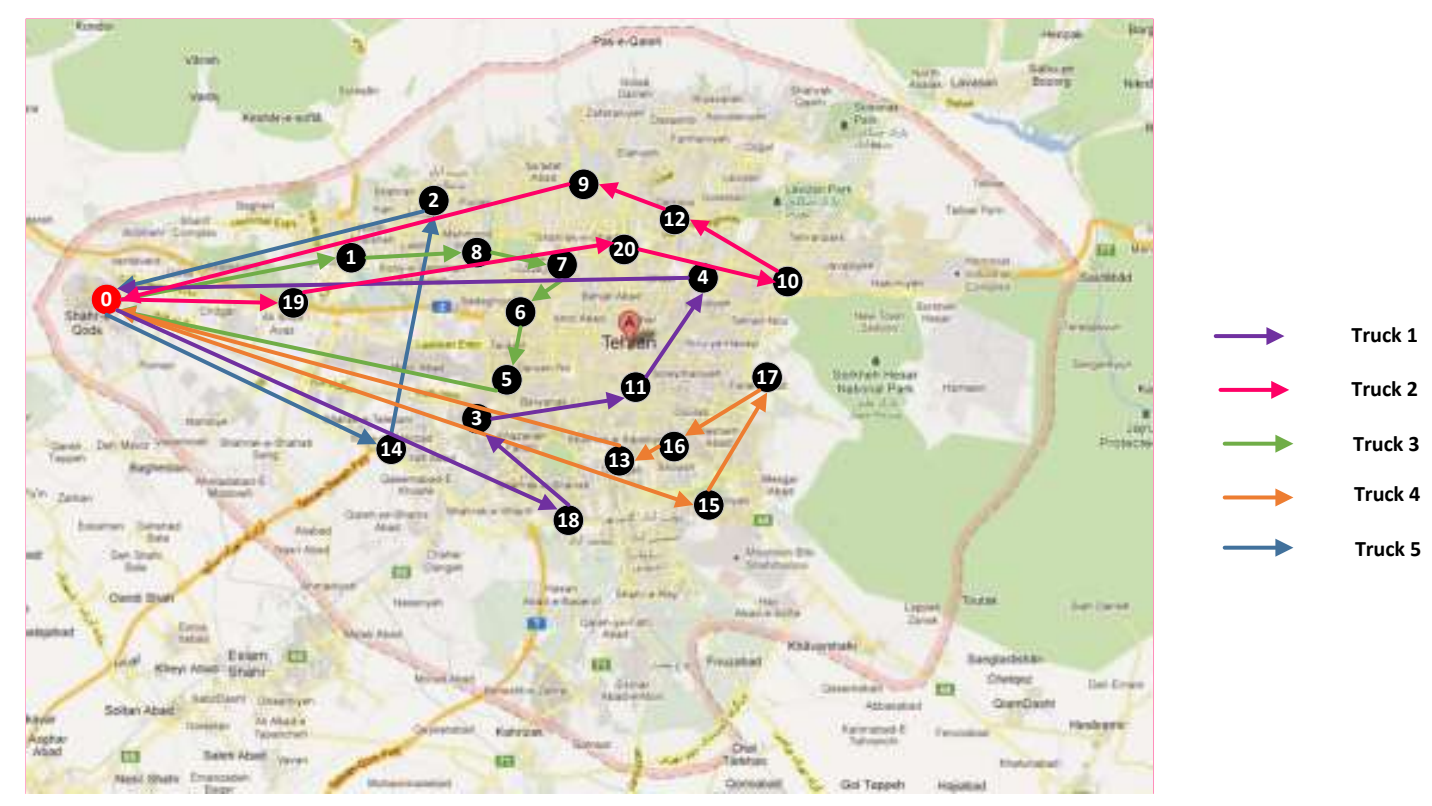

Fig. 10. The routing of vehicles based on case study optimization 


\subsection{The reduced case study}

The experiments for the sensitivity analysis should be conducted by solving an exact method. Since the size of case study is very large to be solved, the number of customers, vehicles (both incoming and outgoing) and 1dock doors are reduced to 9, 3 and 2, respectively. Also, it should be noted that budget is decreased to 300 . Other parameters are as same as mentioned in Table7.

\subsection{The impact of freshness life changes}

To analyze the results of the mathematical modeling, we modified the average freshness life of products. As illustrated in the Fig. 11, by increasing the freshness life of all the products at the same time, the load on the drivers becomes more leveled, and in addition, the costs of earliness (tardiness) increases (decreases) totally.

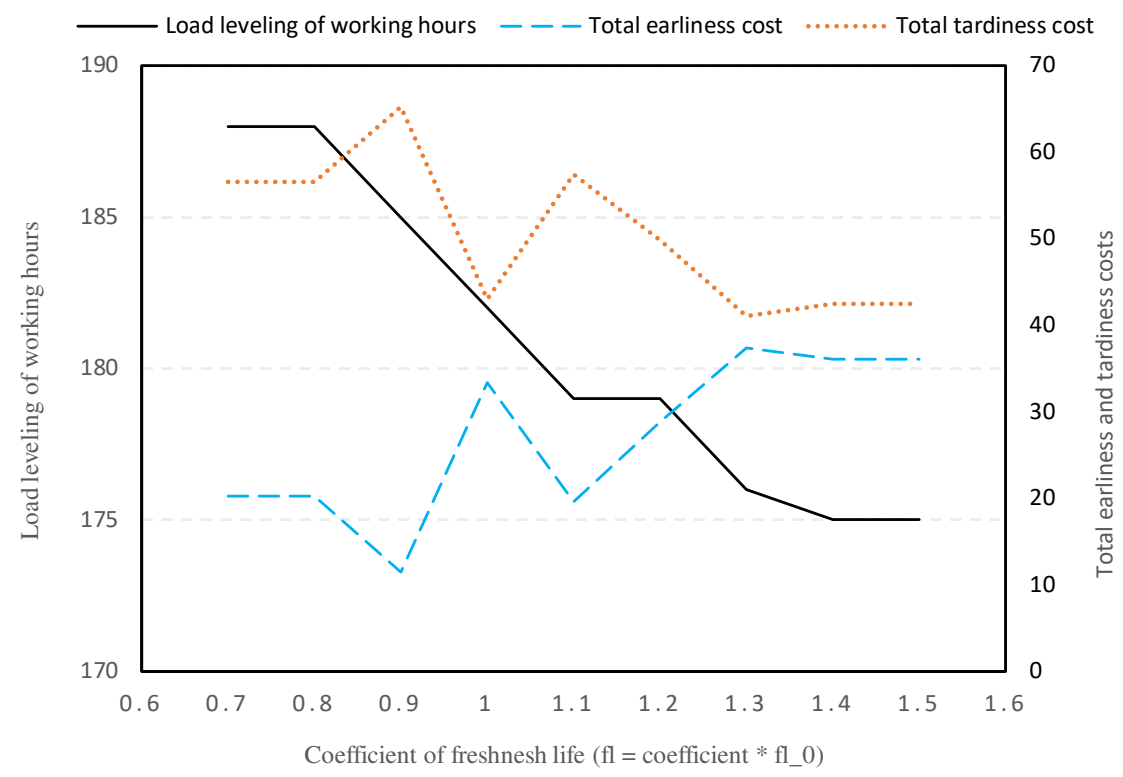

Fig. 11. The impact of freshness life changes on objective function

Fig. 10 shows the effect of changes in freshness life of products on the objective function (fl_0 is given in Table 7 as $f l_{p}$ ). The objective function decreases with increasing product freshness life. With increasing freshness life, solution space is developed, as a consequence the optimal answer is decreased.

\subsection{The impact of budget changes}

The other factor which is modified to show model application is budget. As Fig. 12 shows, changing the budget affect the objective function through budget constraint. With increasing the budget, the fairness of working conditions of the drivers increases. In addition, the average freshness of the delivered products first increases, then decreases, and finally increases. 


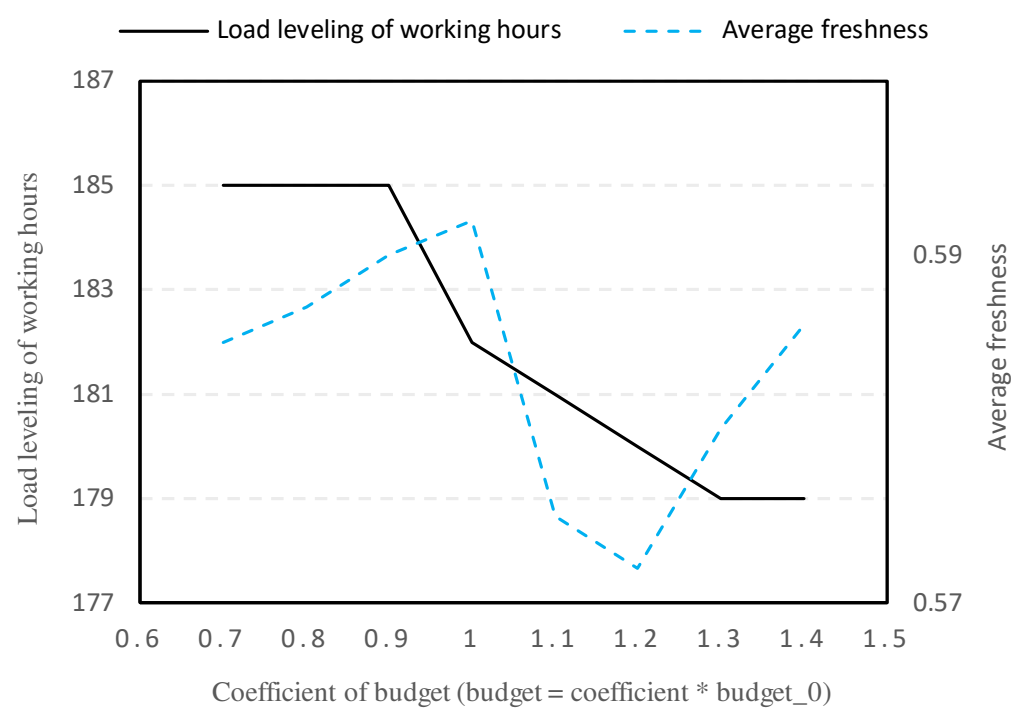

Fig. 12. The impact of budget changes on objective function

\section{Conclusion}

The scheduling and routing problem for perishable product is one of the most important and practical issues in order to reduce costs and delivery time. In this study, cross-dock is considered as a product distribution technique in the supply chain. Since sustainability is a relatively new concept in logistics systems, the model of scheduling and routing of vehicles in a sustainable cross-dock and its solution method were studied. The purpose of scheduling a cross-dock is to determine the sequence and allocation of incoming and outgoing vehicles with the aim of optimizing the goals of the cross-dock management. Therefore, in this research, literature in the field of sustainable vehicle routing was reviewed; then the new sustainable mixed integer model is presented. In the economic dimension of sustainability, there are various costs, including earliness and tardiness penalties, inventory holding and transportation costs. In the environmental dimension, the amount of fuel consumption and consequently the amount of carbon dioxide emissions is limited. In the social dimension, driver satisfaction is examined in order to balancing drivers. Customer satisfaction also considered due to the perishability of the products. In order to solve this model, a GA based on fix and optimize solution is presented. To examine the efficiency of the proposed algorithm, 20 test problems are applied. In these test problems, the CPU time of using the pure mathematical model with the CPLEX solver is higher than the one of MGA algorithm. The case study of Tehran was also examined to show the efficiency of the model and is solved in two cases: 1) all decisions should be made in the GA algorithm and 2) the decision related to the sequence of the order of customers should be optimized in CPLEX. The results show that the second mode is efficient in the long run time. Also, the results of sensitivity analysis show that with decreasing the freshness life of the products, the objective function will be increased. The behaviors of objective function with budget changes is similar to the behavior of the objective function with freshness life changes. This research can be developed in several ways: 1) using other methods to solve the proposed model and comparing the results with the results of the solution method of this study, 2) using other social considerations in the cross-dock operation, 3) solving the problem as a multi-objective problem. 


\section{Author Declarations}

\section{Funding}

"The authors declare that no funds, grants, or other support were received during the preparation of this manuscript."

\section{Competing Interests}

"The authors have no relevant financial or non-financial interests to disclose."

\section{Author Contributions}

"All authors contributed to the study conception and design. Material preparation, data collection and analysis were performed by all authors. The first draft of the manuscript was written by Fatemeh Shahrabi and Mohammad Mahdi Nasiri and all authors commented on previous versions of the manuscript. All authors read and approved the final manuscript."

\section{Data Availability}

"Information on data source is provided as a supplementary material"

\section{Code availability}

"The codes developed for this project are confidential, but may be obtained with contacting the corresponding author. Researchers interested in access to the codes may contact Mohammad Mahdi Nasiriat mmnasiri@ut.ac.ir."

\section{References}

Abad, H. K. E., Vahdani, B., Sharifi, M., \& Etebari, F. (2018). A bi-objective model for pickup and delivery pollution-routing problem with integration and consolidation shipments in cross-docking system. Journal of Cleaner Production.

Abdullahi, H., Reyes-Rubiano, L., Ouelhadj, D., Faulin, J., \& Juan, A. A. (2021). Modelling and multi-criteria analysis of the sustainability dimensions for the green vehicle routing problem. European Journal of Operational Research, 292(1), 143-154.

Afshar-Bakeshloo, M., Mehrabi, A., Safari, H., Maleki, M., \& Jolai, F. (2016). A green vehicle routing problem with customer satisfaction criteria. Journal of Industrial Engineering International, 12(4), 529-544.

Alamatsaz, K., Ahmadi, A., \& Mirzapour Al-e-hashem, S. M. J. (2021). A multiobjective model for the green capacitated location-routing problem considering drivers' satisfaction and time window with uncertain demand. Environmental Science and Pollution Research, 1-20.

Arbabi, H., Nasiri, M. M., \& Bozorgi-Amiri, A. (2021). A hub-and-spoke architecture for a parcel delivery system using the cross-docking distribution strategy. Engineering Optimization, 53(9), 1593-1612.

Bandeira, J. M., Guarnaccia, C., Fernandes, P., \& Coelho, M. C. (2018). Advanced impact integration platform for cooperative road use. International journal of intelligent transportation systems research, 16(1), 1-15.

Bektaş, T., \& Laporte, G. (2011). The Pollution-Routing Problem. Transportation Research Part B: Methodological, 45(8), 1232-1250.

Benjaafar, S., Li, Y., \& Daskin, M. (2012). Carbon footprint and the management of supply chains: Insights from simple models. IEEE transactions on automation science and engineering, 10(1), 99-116.

Brundland, G. (1987). World Commission on Environment and Development. Our Common Future Oxford. In: University Press.. Oxford.

Chan, F. T., Wang, Z., Goswami, A., Singhania, A., \& Tiwari, M. K. (2020). Multi-objective particle swarm optimisation based integrated production inventory routing planning for efficient perishable food logistics operations. International Journal of Production Research, 58(17), 5155-5174. 
Chargui, T., Bekrar, A., Reghioui, M., \& Trentesaux, D. (2020). Scheduling trucks and storage operations in a multiple-door cross-docking terminal considering multiple storage zones. International Journal of Production Research, 1-25.

Ćirović, G., Pamučar, D., \& Božanić, D. (2014). Green logistic vehicle routing problem: Routing light delivery vehicles in urban areas using a neuro-fuzzy model. Expert Systems with Applications, 41(9), 4245-4258.

Govindan, K., Jafarian, A., \& Nourbakhsh, V. (2015). Bi-objective integrating sustainable order allocation and sustainable supply chain network strategic design with stochastic demand using a novel robust hybrid multi-objective metaheuristic. Computers \& Operations Research, 62, 112-130.

Govindan, K., Jafarian, A., \& Nourbakhsh, V. (2019). Designing a sustainable supply chain network integrated with vehicle routing: A comparison of hybrid swarm intelligence metaheuristics. Computers \& Operations Research, 110, 220235.

Hosseini-Nasab, H., \& Lotfalian, P. (2017). Green routing for trucking systems with classification of path types. Journal of cleaner production, 146, 228-233.

Nasiri, M. M., Rahbari, A., Werner, F., \& Karimi, R. (2018). Incorporating supplier selection and order allocation into the vehicle routing and multi-cross-dock scheduling problem. International Journal of Production Research, 56(19), 6527-6552.

Peng, X.-s., Ji, S.-f., \& Ji, T.-t. (2020). Promoting sustainability of the integrated production-inventory-distribution system through the Physical Internet. International Journal of Production Research, 58(22), 6985-7004.

Qiao, Q., Tao, F., Wu, H., Yu, X., \& Zhang, M. (2020). Optimization of a capacitated vehicle routing problem for sustainable municipal solid waste collection management using the PSO-TS algorithm. International journal of environmental research and public health, 17(6), 2163.

Rahbari, A., Nasiri, M. M., Werner, F., Musavi, M., \& Jolai, F. (2019). The vehicle routing and scheduling problem with crossdocking for perishable products under uncertainty: Two robust bi-objective models. Applied Mathematical Modelling, 70, 605-625.

Rahimi, M., Baboli, A., \& Rekik, Y. (2016). Sustainable inventory routing problem for perishable products by considering reverse logistic. IFAC-PapersOnLine, 49(12), 949-954.

Ramos, T. R. P., Gomes, M. I., \& Barbosa-Póvoa, A. P. (2014). Planning a sustainable reverse logistics system: Balancing costs with environmental and social concerns. Omega, 48, 60-74.

Reyes-Rubiano, L., Calvet, L., Juan, A. A., Faulin, J., \& Bové, L. (2018). A biased-randomized variable neighborhood search for sustainable multi-depot vehicle routing problems. Journal of Heuristics, 1-22.

Rezaei, S., \& Kheirkhah, A. (2018). A comprehensive approach in designing a sustainable closed-loop supply chain network using cross-docking operations. Computational and Mathematical Organization Theory, 24(1), 51-98.

Shahedi, A., Nasiri, M. M., Sangari, M. S., Werner, F., \& Jolai, F. (2021). A Stochastic Multi-Objective Model for a Sustainable Closed-Loop Supply Chain Network Design in the Automotive Industry. Process Integration and Optimization for Sustainability, 1-21.

Shahrabi, F., Tavakkoli-Moghaddam, R., Triki, C., Pahlevani, M., \& Rahimi, Y. (2021). Modelling and solving the bi-objective production-transportation problem with time windows and social sustainability. IMA Journal of Management Mathematics.

Song, B. D., \& Ko, Y. D. (2016). A vehicle routing problem of both refrigerated-and general-type vehicles for perishable food products delivery. Journal of food engineering, 169, 61-71.

Tavakkoli-Moghaddam, R., \& Raziei, Z. (2016). A new bi-objective location-routing-inventory problem with fuzzy demands. IFAC-PapersOnLine, 49(12), 1116-1121.

Tirkolaee, E. B., Goli, A., Faridnia, A., Soltani, M., \& Weber, G.-W. (2020). Multi-objective optimization for the reliable pollution-routing problem with cross-dock selection using Pareto-based algorithms. Journal of Cleaner Production, 276, 122927. 
Yin, P.-Y., \& Chuang, Y.-L. (2016). Adaptive memory artificial bee colony algorithm for green vehicle routing with crossdocking. Applied Mathematical Modelling, 40(21), 9302-9315.

Yin, P.-Y., Lyu, S.-R., \& Chuang, Y.-L. (2016). Cooperative coevolutionary approach for integrated vehicle routing and scheduling using cross-dock buffering. Engineering Applications of Artificial Intelligence, 52, 40-53.

Zhalechian, M., Tavakkoli-Moghaddam, R., Zahiri, B., \& Mohammadi, M. (2016). Sustainable design of a closed-loop location-routing-inventory supply chain network under mixed uncertainty. Transportation Research Part E: Logistics and Transportation Review, 89, 182-214.

Zhu, L., \& Hu, D. (2019). Study on the vehicle routing problem considering congestion and emission factors. International Journal of Production Research, 57(19), 6115-6129. 